\title{
LAPLACE TRANSFORM AND Z-TRANSFORM: UNIFICATION AND EXTENSION *
}

\author{
MARTIN BOHNER ${ }^{\dagger}$ AND ALLAN PETERSON ${ }^{\ddagger}$
}

\begin{abstract}
We introduce the Laplace transform for an arbitrary time scale. Two particular choices of time scales, namely the reals and the integers, yield the concepts of the classical Laplace transform and of the classical Z-transform. Other choices of time scales yield new concepts of our Laplace transform, which can be applied to find solutions of higher order linear dynamic equations with constant coeffficients. We present several useful properties of our Laplace transform and offer formulas for the Laplace transforms of many elementary functions, among them results for the convolution of two functions on a time scale, which is introduced in this paper as well.
\end{abstract}

1. Introduction. A time scale is a nonempty closed subset of the reals. In this paper the symbol $\mathbb{T}$ denotes a time scale which is unbounded above and contains zero.

Definition 1.1. We define the Laplace Transform of a regulated function $x$ : $\mathbb{T} \rightarrow \mathbb{C}$ by

$$
\mathcal{L}\{x\}(z)=\int_{0}^{\infty} x(t) \mathrm{e}_{\ominus z}(\sigma(t)) \Delta t \quad \text { for } \quad z \in \mathcal{D}\{x\},
$$

where $\mathcal{D}\{x\}$ consists of all $z \in \mathbb{C}$ for which the improper integral exists and for which $1+\mu(t) z \neq 0$ for all $t \in \mathbb{T}$.

All expressions occurring in Definition 1.1 which are related to the time scales calculus are introduced in Section 2 below. At this point we want the reader only to know that

- if $\mathbb{T}=\mathbb{R}$, then $\sigma(t)=t, \mu(t) \equiv 0, \ominus z=-z, \mathrm{e}_{\alpha}(t)=e^{\alpha t}$, and $\int_{0}^{\infty} f(t) \Delta t=$ $\int_{0}^{\infty} f(t) \mathrm{d} t$

- if $\mathbb{T}=\mathbb{Z}$, then $\sigma(t)=t+1, \mu(t) \equiv 1, \ominus z=-\frac{z}{1+z}, \mathrm{e}_{\alpha}(t)=(1+\alpha)^{t}$, and $\int_{0}^{\infty} f(t) \Delta t=\sum_{t=0}^{\infty} f(t)$

Hence, clearly, if $\mathbb{T}=\mathbb{R}$, our Laplace transform is the classical Laplace transform, while if $\mathbb{T}=\mathbb{Z}$, our Laplace transform is

$$
\mathcal{L}\{x\}(z)=\sum_{t=0}^{\infty} x(t)\left(1-\frac{z}{1+z}\right)^{t+1}=\sum_{t=0}^{\infty} \frac{x(t)}{(z+1)^{t+1}}=\frac{\mathcal{Z}\{x\}(z+1)}{z+1},
$$

where $\mathcal{Z}\{x\}(z)=\sum_{t=0}^{\infty} x(t) / z^{t}$ is the classical Z-transform (see e.g., [11, Section 3.7]). These remarks explain the "unification" property of our Laplace transform, which is also shared by Hilger's Laplace transform (which is different from ours) introduced in [10, Section 6] (but with formulas much more complicated than ours). However, his Laplace transform may not work unless the time scale is either $\mathbb{R}$ or $h \mathbb{Z}=\{h k \mid k \in \mathbb{Z}\}, h>0$, while our Laplace transform is defined for an arbitrary time scale $\mathbb{T}$. E.g., when applying our Laplace transform to the particular time scale $\left\{q^{k} \mid k \in \mathbb{Z}\right\} \cup\{0\}, q>1$, we can use it to solve higher order linear $q$-difference equations

\footnotetext{
*Received January 31, 2001; accepted for publication November 16, 2001.

$\dagger$ University of Missouri-Rolla, Department of Mathematics and Statistics, Rolla, MO 65409-0020, USA (bohner@umr.edu).

$\ddagger$ University of Nebraska-Lincoln, Department of Mathematics and Statistics, Lincoln, NE 685880323, USA (apeterso@math.unl.edu).
} 
TABLE 1.1

Laplace Transforms

\begin{tabular}{|c||c|c|c|c|c|c|c|c|}
\hline$x$ & 1 & $h_{k}$ & $\mathrm{e}_{\alpha}$ & $u_{a}$ & $\cosh _{\alpha}$ & $\sinh _{\alpha}$ & $\cos _{\alpha}$ & $\sin _{\alpha}$ \\
\hline $\mathcal{L}\{x\}(z)$ & $\frac{1}{z}$ & $\frac{1}{z^{k+1}}$ & $\frac{1}{z-\alpha}$ & $\frac{\mathrm{e}_{\ominus z}(a)}{z}$ & $\frac{z}{z^{2}-\alpha^{2}}$ & $\frac{\alpha}{z^{2}-\alpha^{2}}$ & $\frac{z}{z^{2}+\alpha^{2}}$ & $\frac{\alpha}{z^{2}+\alpha^{2}}$ \\
\hline$x$ & $\mathrm{e}_{\alpha} \sin _{\beta /(1+\mu \alpha)}$ & $\mathrm{e}_{\alpha} \cos _{\beta /(1+\mu \alpha)}$ & $\mathrm{e}_{\alpha} \sinh _{\beta /(1+\mu \alpha)}$ & $\mathrm{e}_{\alpha} \cosh _{\beta /(1+\mu \alpha)}$ \\
\hline $\mathcal{L}\{x\}(z)$ & $\frac{\beta}{(z-\alpha)^{2}+\beta^{2}}$ & $\frac{z-\alpha}{(z-\alpha)^{2}+\beta^{2}}$ & $\frac{\beta}{(z-\alpha)^{2}-\beta^{2}}$ & $\frac{z-\alpha}{(z-\alpha)^{2}-\beta^{2}}$ \\
\hline
\end{tabular}

with constant coefficients. The fact that this "extension" property is achieved by the Laplace transform as introduced in Definition 1.1 above, emphasizes that our definition is the most "natural" one.

The set up of this paper is as follows: Section 2 contains a very brief introduction into the time scales calculus as well as some preliminaries on the exponential function, which occurs in the definition of the Laplace transform in (1.1). In Section 3 we prove the following two theorems.

ThEOREM 1.2. If $x: \mathbb{T} \rightarrow \mathbb{C}$ is such that $x^{\Delta}$ is regulated, then

$$
\mathcal{L}\left\{x^{\Delta}\right\}(z)=z \mathcal{L}\{x\}(z)-x(0)
$$

for all $z \in \mathcal{D}\{x\}$ such that $\lim _{t \rightarrow \infty}\left\{x(t) \mathrm{e}_{\ominus z}(t)\right\}=0$.

Again, at this point it is sufficient to know that $x^{\Delta}=x^{\prime}$ (the usual derivative) if $\mathbb{T}=\mathbb{R}$ and that $x^{\Delta}=\Delta x$ (the usual forward difference) if $\mathbb{T}=\mathbb{Z}$. (Regulated means continuous for $\mathbb{T}=\mathbb{R}$ and is no restriction for $\mathbb{T}=\mathbb{Z}$.)

TheOREM 1.3. If $x: \mathbb{T} \rightarrow \mathbb{C}$ is regulated and $X(t)=\int_{0}^{t} x(\tau) \Delta \tau$ for $t \in \mathbb{T}$, then

$$
\mathcal{L}\{X\}(z)=\frac{1}{z} \mathcal{L}\{x\}(z)
$$

for all $z \in \mathcal{D}\{x\} \backslash\{0\}$ such that $\lim _{t \rightarrow \infty}\left\{X(t) \mathrm{e}_{\ominus z}(t)\right\}=0$.

Next, in Section 4, we calculate the Laplace transforms of several important functions, among them the generalized exponential, hyperbolic, trigonometric, and polynomial functions. Table 1.1 shows Laplace transforms of those elementary functions; for the notation we refer to Section 4. It is also remarkable that we obtain "universal" formulas which are independent of the underlying time scales structure (and this is not the case in [10]). This fact even might be an indication to suppose that the classical Z-transform is not the "most natural" one, but that the modified Z-transform defined by $\tilde{\mathcal{Z}}\{x\}(z)=\mathcal{Z}\{x\}(z+1) /(z+1)$ is. One "pragmatic" argument for this last statement is that with our Laplace transform one only has to "know one table" instead of two or more. Finally, in Section 5, we make an attempt to introduce the convolution of two functions defined on the same time scale. This might seem to be an unsuccessful project as the difference of two elements in $\mathbb{T}$ is not necessarily back in $\mathbb{T}$ (unless $\mathbb{T}=\mathbb{R}$ or $\mathbb{T}=h \mathbb{Z}$ ), but we illustrate a way of avoiding this dilemma and introduce convolutions of elementary functions with an arbitrary regulated function. Then we are able to prove the following result.

TheOREM 1.4 (Convolution Theorem). Let $f: \mathbb{T} \rightarrow \mathbb{C}$ be a generalized exponential, hyperbolic, trigonometric, or polynomial function, and let $g: \mathbb{T} \rightarrow \mathbb{C}$ be regulated. Then, subject to a certain limit condition,

$$
\mathcal{L}\{f * g\}=\mathcal{L}\{f\} \cdot \mathcal{L}\{g\} \quad \text { on } \quad \mathcal{D}\{f * g\} .
$$


2. Preliminaries. The calculus on measure chains (and a time scale is a special case of a measure chain) was introduced by Stefan Hilger in [9]. It serves to unify continuous and discrete analysis and to extend those areas to "in between" cases. In this section we will only give a brief overview of the calculus as it is needed for the rest of this paper; for further reading in this relatively new area of research we refer to $[1-9]$.

Again, in this paper $\emptyset \neq \mathbb{T} \subset \mathbb{R}$ denotes a closed set which is unbounded above and which contains 0 . The (forward) jump operator $\sigma$ and the graininess $\mu$ are defined by $\sigma(t)=\inf \{s \in \mathbb{T}: s>t\}$ and $\mu(t)=\sigma(t)-t, t \in \mathbb{T}$, respectively. For a function $f: \mathbb{T} \rightarrow \mathbb{C}$ we define the derivative $f^{\Delta}(t)$ to be the number, if it exists, with the property that for all $\varepsilon>0$ there exists a neighborhood $U$ of $t$ such that $\left|f(\sigma(t))-f(s)-f^{\Delta}(t)(\sigma(t)-s)\right| \leq \varepsilon|\sigma(t)-s|$ for all $s \in U$, and this then defines a function $f^{\Delta}: \mathbb{T} \rightarrow \mathbb{C}$. Three important formulas are

$$
f^{\sigma}=f+\mu f^{\Delta}, \quad(f g)^{\Delta}=f^{\Delta} g+f^{\sigma} g^{\Delta}, \quad\left(\frac{f}{g}\right)^{\Delta}=\frac{f^{\Delta} g-f g^{\Delta}}{g g^{\sigma}},
$$

where $f^{\sigma}:=f \circ \sigma$. A function $f$ defined on $\mathbb{T}$ is called regulated if its right-sided limits exist (and are finite) at points $t \in \mathbb{T}$ with $\sigma(t)=t$ and if its left-sided limits exist (and are finite) at points $t \in \mathbb{T}$ with $\sup \{s \in \mathbb{T}: s<t\}=t$. Hilger's main existence theorem [4, Theorem 6] says that regulated functions possess pre-antiderivatives, and using such an antiderivative $F$ of $f$, i.e., a continuous $F$ with $F^{\Delta}=f$ on a set $D$ where $\mathbb{T} \backslash D$ is countable and does not contain any points $t$ with $\sigma(t)>t$, it is possible to define $\int_{a}^{b} f(t) \Delta t$ by $F(b)-F(a)$ whenever $a, b \in \mathbb{T}$. Next, a function $f$ defined on $\mathbb{T}$ is called regressive if $1+\mu(t) f(t) \neq 0$ for all $t \in \mathbb{T}$. A standard result $[4$, Theorem 8 ] is that the initial value problem

$$
y^{\Delta}=p(t) y, \quad y\left(t_{0}\right)=1
$$

has a unique solution provided $t_{0} \in \mathbb{T}$ and $p$ is regulated and regressive. This unique solution is denoted by $\mathrm{e}_{p}\left(\cdot, t_{0}\right)$. In this paper we will also write $\mathrm{e}_{p}(t)=\mathrm{e}_{p}(t, 0)$. Some useful formulas are

$$
\mathrm{e}_{p}^{\sigma}=(1+\mu p) \mathrm{e}_{p}, \quad \mathrm{e}_{p} \mathrm{e}_{q}=\mathrm{e}_{p \oplus q}, \quad \frac{\mathrm{e}_{p}}{\mathrm{e}_{q}}=\mathrm{e}_{p \ominus q},
$$

where $p \oplus q:=p+q+\mu p q$ and $p \ominus q=(p-q) /(1+\mu q)$. We remark that the set of all regulated and regressive functions is an Abelian group under the addition $\oplus$.

3. Laplace Transform. After having studied the preceding Section 2, the reader may now fully understand our Definition 1.1. Of course linearity of $\mathcal{L}$ (i.e., $\mathcal{L}\{\alpha f+\beta g\}=\alpha \mathcal{L}\{f\}+\beta \mathcal{L}\{g\})$ is now obvious. To derive further results on the Laplace transform, the following auxiliary result is crucial.

LemMa 3.1. If $z \in \mathbb{C}$ is regressive, then

$$
\mathrm{e}_{\ominus z}^{\sigma}=\frac{\mathrm{e}_{\ominus z}}{1+\mu z}
$$

Proof. By the first property mentioned in (2.1) (or (2.2)), the short calculation

$$
\mathrm{e}_{\ominus z}^{\sigma}=[1+\mu(\ominus z)] \mathrm{e}_{\ominus z}=\left[1-\mu \frac{z}{1+\mu z}\right] \mathrm{e}_{\ominus z}=\frac{\mathrm{e}_{\ominus z}}{1+\mu z}
$$


shows the correctness of our claim.

EXAmple 3.2. We now shall find the Laplace transform of $x(t) \equiv 1$. Consider

$\int_{0}^{\infty} \mathrm{e}_{\ominus z}(\sigma(t)) \Delta t=\int_{0}^{\infty} \frac{\mathrm{e}_{\ominus z}(t)}{1+\mu(t) z} \Delta t=\frac{1}{-z} \int_{0}^{\infty}(\ominus z)(t) \mathrm{e}_{\ominus z}(t) \Delta t=\frac{1}{-z} \int_{0}^{\infty} \mathrm{e}_{\ominus z}^{\Delta}(t) \Delta t$,

where we assume $z \neq 0$ is regressive, and where we applied Lemma 3.1 for the first equal sign. Hence $\mathcal{D}\{1\}$ consists of all regressive $z \in \mathbb{C} \backslash\{0\}$ for which $\lim _{t \rightarrow \infty} \mathrm{e}_{\ominus z}(t)$ exists, and for those $z \in \mathcal{D}\{1\}$ with $\lim _{t \rightarrow \infty} \mathrm{e}_{\ominus z}(t)=0$ (e.g., if there exists $c<0$ with $1+\mu(t) c>0$ and $(\ominus z)(t) \leq c$ for all $t \in \mathbb{T}$; see $[9,10]$ and $[5])$ we have

$$
\mathcal{L}\{1\}(z)=\frac{1}{z} .
$$

Proof of Theorem 1.2. We assume that $\lim _{t \rightarrow \infty}\left\{x(t) \mathrm{e}_{\ominus z}(t)\right\}=0$ holds and use the product rule as presented in (2.1) to obtain

$$
\begin{aligned}
\int_{0}^{\infty} x^{\Delta}(t) \mathrm{e}_{\ominus z}(\sigma(t)) \Delta t & =\int_{0}^{\infty}\left\{\left(x \mathrm{e}_{\ominus z}\right)^{\Delta}(t)-x(t) \mathrm{e}_{\ominus z}^{\Delta}(t)\right\} \Delta t \\
& =-x(0)-\int_{0}^{\infty} x(t)(\ominus z)(t) \mathrm{e}_{\ominus z}(t) \Delta t \\
& =-x(0)+z \int_{0}^{\infty} x(t) \frac{\mathrm{e}_{\ominus z}(t)}{1+\mu(t) z} \Delta t \\
& =-x(0)+z \int_{0}^{\infty} x(t) \mathrm{e}_{\ominus z}(\sigma(t)) \Delta t
\end{aligned}
$$

where we have used again Lemma 3.1 for the last equal sign. Thus, if $z \in \mathcal{D}\{x\}$, then $z \in \mathcal{D}\left\{x^{\Delta}\right\}$, too, and formula (1.2) follows.

EXAmple 3.3. Using Theorem 1.2, if $x: \mathbb{T} \rightarrow \mathbb{C}$ is such that $x^{\Delta \Delta}$ is regulated, then

$$
\mathcal{L}\left\{x^{\Delta \Delta}\right\}(z)=z^{2} \mathcal{L}\{x\}(z)-z x(0)-x^{\Delta}(0)
$$

for all $z \in \mathcal{D}\{x\}$ with $\lim _{t \rightarrow \infty}\left\{x(t) e_{\ominus z}(t)\right\}=\lim _{t \rightarrow \infty}\left\{x^{\Delta}(t) e_{\ominus z}(t)\right\}=0$. Corresponding formulas for higher order derivatives can be derived similarly.

Proof of Theorem 1.3. This result follows now immediately by applying Theorem 1.2 to $X$. Note that $X^{\Delta}=x$ and $X(0)=0$ according to certain remarks in Section 2. $\square$

4. Examples and Applications. In this section we verify some of the formulas given in Table 1.1 and illustrate with an example how our Laplace transform can be used in finding solutions of higher order linear dynamic equations with constant coefficients on an arbitrary time scale.

EXAMPLE 4.1. Let $\alpha \in \mathbb{C}$ be regressive (i.e., either $\alpha=0$ or $-1 / \alpha \neq \mu(t)$ for all $t \in \mathbb{T}$ ). We shall now find the Laplace transform of $\mathrm{e}_{\alpha}$. Assuming $z \neq \alpha$, we apply (2.2) and Lemma 3.1 to obtain

$$
\int_{0}^{\infty} \mathrm{e}_{\alpha}(t) \mathrm{e}_{\ominus z}(\sigma(t)) \Delta t=\int_{0}^{\infty} \mathrm{e}_{\alpha}(t) \frac{\mathrm{e}_{\ominus z}(t)}{1+\mu(t) z} \Delta t
$$




$$
\begin{aligned}
& =\int_{0}^{\infty} \frac{\mathrm{e}_{\alpha \ominus z}(t)}{1+\mu(t) z} \Delta t \\
& =\frac{1}{\alpha-z} \int_{0}^{\infty} \frac{\alpha-z}{1+\mu(t) z} \mathrm{e}_{\alpha \ominus z}(t) \Delta t \\
& =\frac{1}{\alpha-z} \int_{0}^{\infty}(\alpha \ominus z)(t) \mathrm{e}_{\alpha \ominus z}(t) \Delta t \\
& =\frac{1}{\alpha-z} \int_{0}^{\infty} \mathrm{e}_{\alpha \ominus z}^{\Delta}(t) \Delta t
\end{aligned}
$$

Thus the formula given in Table 1.1 holds provided $\lim _{t \rightarrow \infty} \mathrm{e}_{\alpha \ominus z}(t)=0$.

EXAMPLE 4.2. If $-\mu \alpha^{2}$ is regressive (i.e., $\alpha$ and $-\alpha$ are regressive), then we define the hyperbolic functions

$$
\cosh _{\alpha}=\frac{\mathrm{e}_{\alpha}+\mathrm{e}_{-\alpha}}{2} \text { and } \sinh _{\alpha}=\frac{\mathrm{e}_{\alpha}-\mathrm{e}_{-\alpha}}{2}
$$

(as functions of two variables; but as before we also will use e.g., $\cosh _{\alpha}(t)=$ $\left.\cosh _{\alpha}(t, 0)\right)$. By linearity and under suitable assumptions we have e.g.,

$$
\mathcal{L}\left\{\cosh _{\alpha}\right\}(z)=\frac{\frac{1}{z-\alpha}+\frac{1}{z+\alpha}}{2}=\frac{z}{z^{2}-\alpha^{2}} .
$$

The formula for $\sinh _{\alpha}$ and those for the trigonometric functions defined by (assuming $\mu \alpha^{2}$ is regressive, i.e., $i \alpha$ and $-i \alpha$ are regressive)

$$
\cos _{\alpha}=\frac{\mathrm{e}_{i \alpha}+\mathrm{e}_{-i \alpha}}{2} \text { and } \sin _{\alpha}=\frac{\mathrm{e}_{i \alpha}-\mathrm{e}_{-i \alpha}}{2 i},
$$

are easy to verify. (Note, however, that the above special functions do not satisfy fundamental identities such as $\cos ^{2}+\sin ^{2}=\cosh ^{2}-\sinh ^{2}=1$, while those defined in [10] do satisfy such identities; see also [7].)

EXAMPLE 4.3. In [1, Section 3], the generalized polynomials

$$
h_{0}(t, s) \equiv 1, \quad h_{k+1}(t, s)=\int_{s}^{t} h_{k}(\tau, s) \Delta \tau \quad \text { for } \quad k \in \mathbb{N}_{0}
$$

are introduced. Let $h_{k}(t)=h_{k}(t, 0)$. We claim that

$$
\mathcal{L}\left\{h_{k}\right\}(z)=\frac{1}{z^{k}} \quad \text { if } \quad z \text { is regressive with } \lim _{t \rightarrow \infty}\left\{h_{k}(t) \mathrm{e}_{\ominus z}(t)\right\}=0
$$

holds for all $k \in \mathbb{N}_{0}$. By Example 3.2, (4.1) holds for $k=0$. Assuming that (4.1) holds for $k=m \in \mathbb{N}_{0}$, we find that

$$
\mathcal{L}\left\{h_{m+1}\right\}(z)=\frac{1}{z} \mathcal{L}\left\{h_{m}\right\}(z)=\frac{1}{z} \frac{1}{z^{m}}=\frac{1}{z^{m+1}}
$$

provided $z \neq 0$ is regressive and satisfies $\lim _{t \rightarrow \infty}\left\{h_{m+1}(t) \mathrm{e}_{\ominus z}(t)\right\}=0$ (which implies $\left.\lim _{t \rightarrow \infty}\left\{h_{m}(t) \mathrm{e}_{\ominus z}(t)\right\}=0\right)$, where we used the relation

$$
h_{m+1}(t)=\int_{0}^{t} h_{m}(\tau) \Delta \tau
$$


and applied Theorem 1.3. Hence (4.1) holds for $k=m+1$, and this establishes our original claim.

EXAMPLE 4.4. Let $a \in \mathbb{T}, a>0$, and $u_{a}(t)=0$ for $t \in \mathbb{T}, t<a$, while $u_{a}(t)=1$ for $t \in \mathbb{T}, t \geq a$. We find

$$
\int_{0}^{\infty} u_{a}(t) \mathrm{e}_{\ominus z}(\sigma(t)) \Delta t=\int_{a}^{\infty} \mathrm{e}_{\ominus z}(\sigma(t)) \Delta t=\frac{\mathrm{e}_{\ominus z}(a)}{z}
$$

provided $z$ is regressive and satisfies $\lim _{t \rightarrow \infty} \mathrm{e}_{\ominus z}(t)=0$ (for the last equal sign compare with the calculation in Example 3.2).

EXAMPLE 4.5. As a specific example how the Laplace transform can be applied to find solutions of initial value problems, consider

$$
y^{\Delta \Delta \Delta}+y^{\Delta}=\mathrm{e}_{1}(t), \quad y(0)=y^{\Delta}(0)=y^{\Delta \Delta}(0)=0 .
$$

Take the Laplace transform on both sides to arrive at

$$
z^{3} \mathcal{L}\{y\}(z)+z \mathcal{L}\{y\}(z)=\frac{1}{z-1}
$$

and hence

$$
\mathcal{L}\{y\}(z)=\frac{1}{\left(z^{3}+z\right)(z-1)}=-\frac{1}{z}+\frac{\frac{1}{2}}{z-1}+\frac{\frac{1}{2} z-\frac{1}{2}}{z^{2}+1}
$$

so that $y(t)=-1+\frac{1}{2} \mathrm{e}_{1}(t)+\frac{1}{2} \cos _{1}(t)-\frac{1}{2} \sin _{1}(t)$, and this $y$ is easily verified to be the solution of (4.2). E.g., if $\mathbb{T}^{2}=\mathbb{Z}^{2}=\left\{k^{2} \mid k \in \mathbb{Z}\right\}$, then $y$ can be determined using

$$
\mathrm{e}_{1}(t)=(\sqrt{t}) ! 2^{\sqrt{t}}, \quad \mathrm{e}_{i}(t)=\prod_{n=1}^{\sqrt{t}}[1+(2 n-1) i], \quad \text { and } \quad \mathrm{e}_{-i}(t)=\prod_{n=1}^{\sqrt{t}}[1-(2 n-1) i]
$$

5. Convolutions. Suppose $\alpha \in \mathbb{C}$ is regressive and $g: \mathbb{T} \rightarrow \mathbb{C}$ is regulated. By $[7$, Theorem 4.2], the unique solution of the initial value problem

$$
y^{\Delta}-\alpha y=g(t), \quad y(0)=0
$$

is given by $y(t)=\int_{0}^{t} \mathrm{e}_{\alpha}(t, \sigma(\tau)) g(\tau) \Delta \tau$. This motivates the following definition.

Definition 5.1. For regressive $\alpha \in \mathbb{C}$ and regulated $g: \mathbb{T} \rightarrow \mathbb{C}$ we define the convolution of $\mathrm{e}_{\alpha}$ and $g$ by

$$
\left(\mathrm{e}_{\alpha} * g\right)(t)=\int_{0}^{t} \mathrm{e}_{\alpha}(t, \sigma(\tau)) g(\tau) \Delta \tau
$$

Proof of Theorem 1.4 (for $f=\mathrm{e}_{\alpha}$ ). With $y=f * g$ we have by Theorem 1.2

$$
\mathcal{L}\{g\}(z)=\mathcal{L}\left\{y^{\Delta}-\alpha y\right\}(z)=z \mathcal{L}\{y\}(z)-\alpha \mathcal{L}\{y\}(z)=(z-\alpha) \mathcal{L}\{y\}(z)
$$

so that, provided $z \neq \alpha$,

$$
\mathcal{L}\{f * g\}(z)=\mathcal{L}\{y\}(z)=\frac{1}{z-\alpha} \mathcal{L}\{g\}(z)=\mathcal{L}\left\{\mathrm{e}_{\alpha}\right\}(z) \mathcal{L}\{g\}(z)=\mathcal{L}\{f\}(z) \mathcal{L}\{g\}(z)
$$


if $z \in \mathcal{D}\{y\}$ such that $\lim _{t \rightarrow \infty} \mathrm{e}_{\alpha \ominus z}(t)=0$.

Convolutions of hyperbolic and trigonometric functions (and also of generalized polynomials) with another regulated function $g$ are now defined in a similar manner as in (5.1):

$$
\left(\cosh _{\alpha} * g\right)(t)=\int_{0}^{t} \cosh _{\alpha}(t, \sigma(\tau)) g(\tau) \Delta \tau
$$

and so on. It is easy to verify that Theorem 1.4 holds for those cases as well (use induction for the generalized polynomials and linearity together with what we already have shown for the other cases). Observe that we avoid the occurrence of $t-\tau$ in those definitions, which would be ambiguous since as pointed out earlier a general time scale need not contain the difference of two of its elements. An open question remains on how to define convolution with two arbitrary regulated functions. However, for the purpose of solving higher order linear dynamic equations with constant coefficients on time scales, the material presented in this section is sufficient.

\section{REFERENCES}

[1] R.P. Agarwal And M. Bohner, Basic calculus on time scales and some of its applications, Results Math., 35 (1-2) (1999), pp. 3-22.

[2] R.P. Agarwal, M. Bohner, D. O'Regan and A. Peterson, Dynamic equations on time scales: A survey, J. Comput. Appl. Math., 141 (1-2) 2002, pp. 1-26. Special Issue on "Dynamic Equations on Time Scales", edited by R.P. Agarwal, M. Bohner, and D. O'Regan.

[3] E. Akin, L. Erbe, B. Kaymakçalan and A. Peterson, Oscillation results for a dynamic equation on a time scale, J. Differ. Equations Appl., 7 (6)(2001), pp. 793-810.

[4] B. Aulbach And S. Hilger, Linear dynamic processes with inhomogeneous time scale. In Nonlinear Dynamics and Quantum Dynamical Systems (Gaussig, 1990), vol. 59 of Math. Res., pp. 9-20. Akademie Verlag, Berlin, 1990.

[5] M. Bohner And R. Hering, Perturbations of dynamic equations, J. Difference Equ. Appl., 8 (4)(2002), pp. 295-305.

[6] M. Bohner AND D.A. Lutz, Asymptotic behavior of dynamic equations on time scales, J. Differ. Equations Appl., 7 (1) (2001), pp. 21-50.

[7] M. Bohner And A. Peterson, First and second order linear dynamic equations on measure chains, J. Differ. Equations Appl., 7 (6)(2001), pp. 767-792.

[8] L. Erbe And A. Peterson, Green's functions and comparison theorems for differential equations on measure chains, Dynam. Contin. Discrete Impuls. Systems, 6(1)(1999), pp. 121137.

[9] S. Hilger, Analysis on measure chains - a unified approach to continuous and discrete calculus, Results Math., 18 (1990), pp. 18-56.

[10] S. Hilger, Special functions, Laplace and Fourier transform on measure chains, Dynam. Systems Appl., 8(3-4) (1999), pp. 471-488. Special Issue on "Discrete and Continuous Hamiltonian Systems", edited by R.P. Agarwal and M. Bohner.

[11] W.G. Kelley And A.C. Peterson, Difference Equations: An Introduction with Applications, Academic Press, San Diego, second edition, 2001. 
